\title{
Truncation Formulas for Invariant Polynomials of Matroids and Geometric Lattices
}

\author{
Relinde Jurrius · Ruud Pellikaan
}

Received: 29 November 2011 / Revised: 6 June 2012 / Accepted: 12 June 2012 / Published online: 21 July 2012

(C) The Author(s) 2012. This article is published with open access at Springerlink.com

\begin{abstract}
This paper considers the truncation of matroids and geometric lattices. It is shown that the truncated matroid of a representable matroid is again representable. Truncation formulas are given for the coboundary and Möbius polynomial of a geometric lattice and the spectrum polynomial of a matroid, generalizing the truncation formula of the rank generating polynomial of a matroid by Britz.
\end{abstract}

Keywords Matroid theory $\cdot$ Geometric lattice $\cdot$ Invariant polynomials

Mathematics Subject Classification (2010) $\quad 05 B 35$

\section{Introduction}

The mathematical meaning of truncation is just as it is in every day life: cutting something off. In the situation of this paper, we will be "cutting off" matroids and geometric lattices, by 'removing' their hyperplanes and bases, respectively. An overview of more possible constructions on matroids and geometric lattices can be found in [4].

There are several polynomials associated to matroids and geometric lattices. Probably the most famous and well studied polynomial is the Tutte polynomial, or its equivalent, the rank generating polynomial. The coboundary polynomial is equivalent to the rank generating polynomial. Polynomials that are not equivalent to the rank generating polynomial include the Möbius polynomial and the spectrum polynomial.

Britz [3] showed that the rank generating function of a truncated matroid is defined by the rank generating function of the matroid itself. It is a natural question to ask if there are similar truncation formulas for other polynomials. We will give truncation formulas for the coboundary, Möbius and spectrum polynomial. The outline of the combinatorial proof for these formulas is the same for all three polynomials.

We will conclude by listing some possible applications and generalizations of the various truncation formulas. The truncations formulas trigger questions related to linear codes, Whitney numbers and Duursma zeta-functions.

R. Jurrius · R. Pellikaan ( $\varangle)$

Department of Mathematics and Computer Science, Eindhoven University of Technology,

P.O. Box 513, 5600 MB, Eindhoven, The Netherlands

e-mail: g.r.pellikaan@tue.nl

R. Jurrius

e-mail: r.p.m.j.jurrius@tue.nl 


\section{Truncation of Matroids and Geometric Lattices}

For an excellent introduction into the topic of matroids; see [14,18]. More about the theory of geometric lattices and the Möbius function can be found in $[1,8,16]$. In [20] the cryptomorphism between matroids and geometric lattices is discussed.

We can define the truncation of a matroid in several equivalent ways, just as we can define a matroid in terms of its independent sets, bases, rank function, flats, circuits, etcetera. We will give some equivalent definitions that are most suitable for our purposes. For a more extensive list; see [4].

Definition 1 The truncation of a matroid $M$ of $\operatorname{rank} r(M)=r \geq 1$ is denoted by $\tau(M)$, it has the same set of elements as $M$ and it has the following equivalent definitions:

- The independent sets of $\tau(M)$ are all the independent sets of $M$, except those of rank $r$.

- The rank function of $\tau(M)$ is given by $r_{\tau(M)}(A)=\min \left\{r_{M}(A), r-1\right\}$.

- The bases of $\tau(M)$ are the independent sets of rank $r-1$ in $M$.

The truncation of a matroid is again a matroid.

Example 2 Consider the uniform matroid $M=U_{r, n}$ on $n$ elements of rank $r \geq 1$. Then $\tau(M)=U_{r-1, n}$.

For a geometric lattice, the definition is more straightforward.

Definition 3 The truncation of a geometric lattice $L$ of $\operatorname{rank} r(L)=r \geq 1$ with partial ordering $\leq$ has as elements all the elements of $L$ except those of rank $r-1$, with the same partial ordering $\leq$. It is denoted by $\tau(L)$.

The truncation of a geometric lattice is again a geometric lattice. Note that the truncations of a matroid and a geometric lattice each have rank $r-1$. For a matroid $M$, we denote by $L(M)$ its associated geometric lattice of flats. Conversely, for a geometric lattice $L$, we denote by $M(L)$ its associated matroid. Now $L(M(L))$ is isomorphic to $L$, and $M(L(M))$ is isomorphic to $M$ if $M$ is a simple matroid, i.e., $M$ has no loops or parallel elements. Furthermore $\tau(M(L))=M(\tau(L))$ and $\tau(L(M))=L(\tau(M))$.

Instead of removing all elements of $\operatorname{rank} r-1$ from a geometric lattice, one might ask what happens if we remove all elements of rank 1, the atoms, from the geometric lattice. Unfortunately, the resulting structure is no longer a geometric lattice. We therefore loosen our definitions to get a class of objects that is closed under this "truncation from below". If we start with a geometric lattice and drop the requirements that it is atomic and that its rank function is semimodular, we get a poset with rank function; see [1].

Definition 4 Let $P$ be a finite poset with partial order $\leq$. Then $P$ is called a poset with a rank function if it has a minimum $0_{P}$, a maximum $1_{P}$ and a rank function $r_{P}$ such that:

- $0_{P} \leq x \leq 1_{P}$ for all $x \in P$,

- $r_{P}\left(0_{P}\right)=0$,

- $r_{P}(x)<r_{P}(y)$ for all $x<y$ in $P$,

- $r_{P}(x)=r_{P}(y)-1$ if $x<y$ and there is no $z$ such that $x<z<y$.

$P_{i}$ is the set of elements $x \in P$ such that $r_{P}(x)=i$. The rank of $P$ is $r_{P}\left(1_{P}\right)$ and is denoted by $r(P)$.

Definition 5 The upper truncation of a poset with rank function $P$ of $\operatorname{rank} r(P)=r \geq 1$ with partial ordering $\leq$ has as elements all the elements of $P$ except those of rank $r-1$, with the same partial ordering $\leq$. It is denoted by $\tau_{+}(L)$. The lower truncation of $P$ has as elements all the elements of $P$ except those of rank 1 , with the same partial ordering $\leq$. It is denoted by $\tau_{-}(L)$.

Again, truncating lowers the rank by 1 , so $\tau_{+}(P)$ and $\tau_{-}(P)$ both have rank $r-1$. Let $L$ be a geometric lattice of rank $r$, and $P(L)$ its associated poset with rank function. Then $P(\tau(L))=\tau_{+}(P(L))$. But it is not true that $\tau_{-}(P(L))$ is the poset of some sort of truncation of the geometric lattice $L$, since it is not always the case that the rank function on $\tau_{-}(P(L))$ is semimodular. 
Example 6 Consider $M=U_{4,4}$ the the uniform matroid on four elements of rank 4. Let $L$ be the lattice of flats of $M$, and $P$ the associated poset of $L$ with rank function. Consider $x=\{1,2\}$ and $y=\{3,4\}$ in $\tau_{-}(P)$. The meet and join of $x$ and $y$ in $\tau_{-}(P)$ are given by $x \wedge y=\emptyset$ and $x \vee y=\{1,2,3,4\}$, respectively. Then $r_{\tau_{-}(P)}(x)=r_{\tau_{-}(P)}(y)=1$ and $r_{\tau_{-}(P)}(x \wedge y)=0$ and $r_{\tau_{-}(P)}(x \vee y)=3$. Hence $\tau_{-}(P)$ is not a geometric lattice.

There is a natural way to extend the lattice $\tau_{-}(L)$ to a geometric lattice, called Dilworth completion. See $[4, \S 7.7],[7,10]$, and $[19, \S 12]$. The result of this competition is referred to as the Dilworth truncation of a lattice. It can be viewed as the smallest geometric lattice that contains $\tau_{-}(L)$. Since our techniques for finding truncation formulas do not apply on the Dilworth truncation, we will not go into the details of its definition.

For every poset $P$, we can define its inverse poset $i(P)$. This poset contains the same elements as $P$, but the order is reversed: $x \geq_{i} y$ in the inverse poset if and only if $x \leq y$ in the original poset. If $P$ is a finite poset, then the Möbius function $\mu_{P}(y, x)$ is well-defined and $\mu_{i(P)}(x, y)=\mu_{P}(y, x)$.

If the poset has a minimum and maximum, then the inverse poset has a maximum and minimum: $0_{P}=1_{i(P)}$ and $1_{P}=0_{i(P)}$. The rank of the poset and its inverse are the same, and the rank function of the inverse poset is given by $r_{i(P)}(x)=r(P)-r_{P}(x)$.

Lower truncation can now be described in terms of upper truncation and inversion: the lower truncation of a poset can be obtained by first inverting the poset, then taking upper truncation, and then inverting back again. On the other hand, we can obtain upper truncation by first inverting, then taking lower truncation, and then inverting back again.

\section{Representation of a Truncated Matroid}

It was shown by Mason $[13, \S 2.4]$ that if a matroid is representable over a field, then the Dilworth truncation of a matroid is again representable over an extension of that field. This question for ordinary truncation is addressed in [4, Prop. 7.4.10]: if $M$ is a matroid that is representable over the field $\mathbb{F}$, then $\tau(M)$ is a representable matroid over a transcendental extension of $\mathbb{F}$. We will give a stronger version of this result.

Example 7 Consider the simplex code $C$ of dimension 3 over the finite field with $q$ elements. This code has length $n=q^{2}+q+1$ and minimum distance $q^{2}$. The associated matroid $M=M_{C}$ is by definition representable. The truncation $\tau(M)$ is the uniform matroid $U_{2, n}$. Now $U_{2, n}$ is representable over $\mathbb{F}_{q}$ if and only if $n$ is at most $q+1$. Therefore $\tau(M)$ is representable over any extension of $\mathbb{F}_{q^{3}}$ but it is not representable over $\mathbb{F}_{q}$ itself.

Theorem 8 Let $M$ be a matroid of rank $k$ on $n$ elements that is representable over a field $\mathbb{F}$.

1. If $\mathbb{F}$ is infinite, then $\tau(M)$ is representable over $\mathbb{F}$.

2. If $\mathbb{F}$ is finite consisting of $q$ elements and $m \geq\left\lceil\log _{q}\left(\left(\begin{array}{c}n \\ k-1\end{array}\right)\right)\right\rceil+1$, then $\tau(M)$ is representable over $\mathbb{F}_{q^{m}}$.

Proof Suppose that $M$ is a matroid of rank $k$ on the set $E=\{1, \ldots, n\}$ and $M$ is represented by a $k \times n$ matrix $G$ of rank $k$ over $\mathbb{F}$. A subset $I$ of $E$ is an independent set of $M$ if and only if the columns of $G$ enumerated by $I$ are independent. Let $C$ be the subspace of $\mathbb{F}^{n}$ generated by the rows of $G$. Then $C$ has dimension $k$, since $G$ has rank $k$. Let $t=n-k$. Let $H\left(j_{1}, \ldots, j_{t}\right)$ be the $t \times t$ matrix obtained from $H$ by taking the columns numbered by $j_{1}, \ldots, j_{t}$, where $1 \leq j_{1}<\cdots<j_{t} \leq n$. Let $\Delta\left(j_{1}, \ldots, j_{t}\right)$ be the determinant of $H\left(j_{1}, \ldots, j_{t}\right)$.

Let $I$ be a basis of $\tau(M)$. Let $J=E \backslash I$. Then $|I|=k-1$ and there exists an $i$ in $J$ such that $I \cup\{i\}$ is a basis of $M$. Hence the columns of $H$ enumerated by $J \backslash\{i\}$ are independent. Let $J=\left\{j_{1}, \ldots, j_{t+1}\right\}$ for some $1 \leq j_{1}<\cdots<j_{t+1} \leq n$. Consider the linear function given by:

$f_{I}\left(X_{1}, \ldots, X_{n}\right)=\sum_{s=1}^{t+1}(-1)^{s} \Delta\left(j_{1}, \ldots, \hat{j}_{s}, \ldots, j_{t+1}\right) X_{j_{s}}$,

where $\left(j_{1}, \ldots, \hat{j}_{s}, \ldots, j_{t+1}\right)$ is the $t$-tuple obtained from $\left(j_{1}, \ldots, j_{s}, \ldots, j_{t+1}\right)$ by deleting the $s$-th element. There exists an $s$ such that $j_{s}=i$ and the corresponding determinant $\Delta\left(j_{1}, \ldots, \hat{j}_{s}, \ldots, j_{t+1}\right)$ is not zero. Hence the above 
equation is not identically zero and defines a hyperplane $\mathcal{H}_{I}$ in $\mathbb{F}^{n}$ with equation $f_{I}\left(X_{1}, \ldots, X_{n}\right)=0$ for every basis $I$ of $\tau(M)$. Consider the product

$$
f\left(X_{1}, \ldots, X_{n}\right)=\prod_{I \text { basis of } M} f_{I}\left(X_{1}, \ldots, X_{n}\right) .
$$

So $f\left(X_{1}, \ldots, X_{n}\right)$ is a non-zero polynomial. If $\mathbb{F}$ is infinite, then this polynomial is not everywhere zero on $\mathbb{F}^{n}$ by [12, V §4 Corollary 3]. Therefore there exists an element $\mathbf{x} \in \mathbb{F}^{n}$ such that $f\left(x_{1}, \ldots, x_{n}\right)$ is not zero.

If $\mathbb{F}$ is finite, then $\mathbb{F}=\mathbb{F}_{q}$ and we assumed $m \geq\left\lceil\log _{q}\left(\left(\begin{array}{c}n \\ k-1\end{array}\right)\right)\right\rceil+1$. So $q^{m}>\left(\begin{array}{c}n \\ k-1\end{array}\right)$. Hence

$\left(q^{m}\right)^{n}>\left(\begin{array}{c}n \\ k-1\end{array}\right)\left(q^{m}\right)^{n-1}$.

The number of basis of $\tau(M)$ is at most $\left(\begin{array}{c}n \\ k-1\end{array}\right)$. Therefore $\mathbb{F}_{q^{m}}^{n}$ has more elements than the union of all hyperplanes $\mathcal{H}_{I}$ with $I$ a basis of $\tau(M)$. So there exists an element $\mathbf{x} \in \mathbb{F}_{q^{m}}^{n}$ which does not lie in this union.

In both cases an $n$-tuple $\mathbf{x}$ is found, possibly over an extension of $\mathbb{F}$ such that $f_{I}\left(x_{1}, \ldots, x_{n}\right)$ is not zero for every basis $I$ of $\tau(M)$. Let $\tilde{H}$ be the $(t+1) \times n$ matrix obtained by adding to $H$ the row $\mathbf{x}$. Then for every basis $I$ of $\tau(M)$ the columns of $\tilde{H}$ enumerated by the complement of $I$ are independent, because the determinant of the corresponding square matrix of size $t+1$ is equal to $f_{I}(\mathbf{x}) \neq 0$.

The conclusion is that the null space of $\tilde{H}$ is a subspace of $C$ of dimension $k-1$ that represents $\tau(M)$.

\section{Truncation and Polynomial Invariants}

For polynomial invariants associated with matroids and geometric lattices, one might ask if we can find the polynomial of their truncation from the polynomial of the original object. The answer is positive for the characteristic polynomial; see [4, p. 149]. For the rank generating function $R_{M}(X, Y)$ of a matroid we have the following Theorem 10, proved by Britz [3, Prop. 15].

Definition 9 Let $M=(E, \mathcal{I})$ be a matroid. The Whitney rank generating function $R_{M}(X, Y)$ is defined by

$R_{M}(X, Y)=\sum_{J \subseteq E} X^{r(E)-r(J)} Y^{|J|-r(J)}$

and the Tutte-Whitney or Tutte polynomial is defined by

$t_{M}(X, Y)=\sum_{J \subseteq E}(X-1)^{r(E)-r(J)}(Y-1)^{|J|-r(J)}$.

Therefore

$t_{M}(X, Y)=R_{M}(X-1, Y-1)$.

Theorem 10 (Truncation formula) Let $M$ be a matroid. Then

$X \cdot R_{\tau(M)}(X, Y)=R_{M}(X, Y)+(X Y-1) \cdot R_{M}(0, Y)$.

In this section, we will adjust the question for a generalization of the characteristic polynomial.

Definition 11 Let $L$ be a geometric lattice. The two-variable characteristic or coboundary polynomial in the variables $S$ and $T$ is given by

$\chi_{L}(S, T)=\sum_{x \in L} \sum_{x \leq y \in L} \mu_{L}(x, y) S^{a_{L}(x)} T^{r(L)-r_{L}(y)}$,

where $a_{L}(x)$ is the number of atoms $a$ in $L$ such that $a \leq x$, and $r_{L}(y)$ is the rank of $y$ in $L$. 
Theorem 12 Let $L$ be a geometric lattice of rank $r \geq 3$. Then

$T \cdot \chi_{\tau(L)}(S, T)=\chi_{L}(S, T)+(T-1) \cdot \chi_{L}(S, 0)$

where $a(L)=a_{L}(1)$ is the number of atoms of $L$.

Proof The identity is a consequence of Theorem 10 using the c (see [5, p. 605] and [6, Thm. II]):

$\chi_{L}(S, T)=(S-1)^{r(L)} \cdot R_{M(L)}\left(\frac{T}{(S-1)}, S-1\right)$.

Since the coboundary polynomial is equivalent to the rank generating function, it is clear that a truncation formula for the coboundary polynomial should exist. However, the definition of the coboundary polynomial makes it possible to find a more general idea behind this truncation formula, that can also be adjusted to other polynomial invariants that are not equivalent to (or determined by) the rank generating polynomial.

Theorem 13 Let $f(S, T)$ be a polynomial invariant of a matroid, geometric lattice, or poset with rank function that has rank $r$ and can be written as

$f(S, T)=\sum_{i=0}^{r} f_{j}(S) T^{r-j}$.

Let $f^{\prime}$ be the polynomial invariant of the truncation of the matroid, geometric lattice, or poset with rank function. So, we can write

$f^{\prime}(S, T)=\sum_{i=0}^{r-1} f_{j}^{\prime}(S) T^{r-1-j}$.

If the coefficients $f_{j}(S)$ and $f_{j}^{\prime}(S)$ are related via

$\left\{\begin{array}{l}f_{j}^{\prime}(S)=f_{j}(S), \quad \text { for } j \leq r-2, \\ f_{j}^{\prime}(S)=f_{j}(S)+f_{j+1}(S), \text { for } j=r-1,\end{array}\right.$

then the following truncation formula holds:

$T \cdot f^{\prime}(S, T)=f(S, T)+(T-1) \cdot f(S, 0)$.

Proof The proof is given by rewriting.

$$
\begin{aligned}
T \cdot f^{\prime}(S, T) & =\sum_{i=0}^{r-1} f_{j}^{\prime}(S) T^{r-j} \\
& =\sum_{i=0}^{r-2} f_{j}(S) T^{r-j}+T \cdot\left(f_{r-1}(S)+f_{r}(S)\right) \\
& =\sum_{i=0}^{r-1} f_{j}(S) T^{r-j}+T \cdot f_{r}(S) \\
& =\sum_{i=0}^{r} f_{j}(S) T^{r-j}-f_{r}(S)+T \cdot f_{r}(S) \\
& =f(S, T)+(T-1) \cdot f(S, 0) .
\end{aligned}
$$

We can use this formula to give a different proof of Theorem 12. 
Proof (Theorem 12) We can not directly apply Theorem 13 to the coboundary polynomial: we will use the following reduced form, where we delete the highest degree terms in $S$. Let $m$ be the number of atoms of $L$ and let

$\bar{\chi}_{L}(S, T)=\chi_{L}(S, T)-S^{m}=\sum_{j=0}^{r} \bar{\chi}_{j}(S) T^{r-j}$,

where

$\bar{\chi}_{j}(S)=\sum_{\substack{a_{L}(x)=i \\ i<m}} \sum_{\substack{x \leq y \\ r_{L}(y)=j}} \mu_{L}(x, y) S^{i}$.

Let the reduced coboundary polynomial of the truncated geometric lattice be given by

$\bar{\chi}^{\prime}(S, T)=\sum_{i=0}^{r-1} \bar{\chi}_{j}^{\prime}(S) T^{r-1-j}$

We need to show the relations of Theorem 13. Notice that if $r_{L}(x) \leq r-2$, then $r_{L}(x)=r_{\tau(L)}(x)$ and $a_{L}(x)=$ $a_{\tau(L)}(x)$. Also, if $r(x), r(y) \leq r-2$, then $\mu_{L}(x, y)=\mu_{\tau(L)}(x, y)$. Therefore, $\bar{\chi}_{j}^{\prime}(S)=\bar{\chi}_{j}(S)$ for $j \leq r-2$.

It is left to show that $\bar{\chi}_{r-1}(S)+\bar{\chi}_{r}(S)=\bar{\chi}_{r-1}^{\prime}(S)$, that is,

$\sum_{\substack{a_{L}(x)=i \\ i<m}} \sum_{\substack{x \leq y \\ r_{L}(y)=r-1}} \mu_{L}(x, y) S^{i}+\sum_{\substack{a_{L}(x)=i \\ i<m}} \mu_{L}\left(x, 1_{L}\right) S^{i}=\sum_{\substack{a_{\tau(L)}(x)=i \\ i<m}} \mu_{\tau(L)}\left(x, y_{\tau(L)}\right) S^{i}$.

Since $a_{L}(x)<m$, we have $r_{L}(x)<r$ in every summation. We split into two parts. If $r_{L}(x)=r-1=r_{\tau(L)}(x)$, then $a_{\tau(L)}(x)=m$, so the right hand side of the summation is 0 . The left hand side of the summation is then equal to

$$
\sum_{\substack{a_{L}(x)=i \\ i<m}} S^{i}(1-1)=0
$$

so this cancels out. Now suppose $r_{L}(x)=r_{\tau(L)}(x) \leq r-2$. We can drop the first summation and the factor $S^{i}$, because they are the same on both sides. So, we need to show that

$\sum_{\substack{x \leq y \\ r_{L}(y)=r-1}} \mu_{L}(x, y)+\mu_{L}\left(x, 1_{L}\right)=\mu_{\tau(L)}\left(x, y_{\tau(L)}\right)$.

We will use the induction formula for the Möbius function: for $x<y$, it holds that

$\sum_{x \leq z \leq y} \mu_{L}(x, z)=\sum_{x \leq z \leq y} \mu_{L}(z, y)=0$.

If we use this on the left hand side, we get

$$
\begin{aligned}
\sum_{\substack{x \leq y \\
r_{L}(y)=r-1}} \mu_{L}(x, y)+\mu_{L}\left(x, 1_{L}\right) & =\sum_{\substack{x \leq y \\
r_{L}(y)=r-1}} \mu_{L}(x, y)-\sum_{x \leq z<1_{L}} \mu_{L}(x, z) \\
& =-\sum_{\substack{x \leq z \\
r_{L}(z) \leq r-2}} \mu_{L}(x, z) .
\end{aligned}
$$

The right hand side is equal to

$$
\begin{aligned}
\mu_{\tau(L)}\left(x, 1_{\tau(L)}\right) & =-\sum_{x \leq z<1_{\tau(L)}} \mu_{\tau(L)}(x, z) \\
= & -\sum_{\substack{x \leq z \\
r_{\tau(L)}(z) \leq r-2}} \mu_{\tau(L)}(x, z) \\
=- & \sum_{\substack{x \leq z \\
r_{L}(z) \leq r-2}} \mu_{L}(x, z)
\end{aligned}
$$


We conclude that $\bar{\chi}_{r-1}(S)+\bar{\chi}_{r}(S)=\bar{\chi}_{r-1}^{\prime}(S)$, so the reduced coboundary polynomial satisfies the conditions in Theorem 13 and therefore has a truncation formula. Since the term $S^{m}$ that we omitted does not change under truncation, we get the truncation formula as is Theorem 12 .

Example 14 The uniform matroid $U_{r, n}$ has rank generating function

$R_{U_{r, n}}(X, Y)=\sum_{i=0}^{r-1}\left(\begin{array}{l}n \\ i\end{array}\right) X^{r-i}+\sum_{i=r}^{n}\left(\begin{array}{l}n \\ i\end{array}\right) Y^{i-r}$,

as follows directly from the definitions. Since the coboundary polynomial is defined by the rank polynomial, we can find the coboundary polynomial of the uniform geometric lattice:

$\chi_{U_{r, n}}(S, T)=\sum_{i=0}^{r-1}\left(\begin{array}{l}n \\ i\end{array}\right)(S-1)^{i} T^{r-i}+\sum_{i=r}^{n}\left(\begin{array}{l}n \\ i\end{array}\right)(S-1)^{i}$.

In this form many terms cancel each other. For example $U_{3,7}$ has coboundary polynomial

$\chi_{U_{3,7}}(S, T)=S^{7}+21 S^{2}(T-1)+7 S\left(T^{2}-6 T+5\right)+\left(T^{3}-7 T^{2}+21 T-15\right)$.

In the form as above, it is straightforward to verify the truncation formula for the coboundary polynomial for arbitrary uniform geometric lattices. In case of the lattice $U_{3,7}$, its truncation $U_{2,7}$ has coboundary polynomial

$\chi_{U_{2,7}}(S, T)=S^{7}+7 S(T-1)+\left(T^{2}-7 T+6\right)$.

\section{Truncation Formula for the Möbius Polynomial}

The combinatorial proof for the truncation formula in Theorem 12 is also applicable to other polynomials. We loosen to the case of posets with rank functions, so we can also address the lower truncation.

Definition 15 Let $P$ be a poset with rank function. The two-variable Möbius polynomial in the variables $S$ and $T$ is given by

$\mu_{P}(S, T)=\sum_{x \in P} \sum_{x \leq y \in P} \mu_{P}(x, y) S^{r_{P}(x)} T^{r(P)-r_{P}(y)}$.

The definition of the Möbius polynomial $\mu_{P}(S, T)$ is similar to the one of the coboundary polynomial $\chi_{L}(S, T)$. However, if $L$ is a geometric lattice and $P$ is the poset with rank function associated with $L$, then $\chi_{L}(S, T)$ and $\mu_{P}(S, T)$ do not determine each other. See $[8, \S 8.6]$ for counterexamples.

Theorem 16 Let $P$ be a poset with rank function of rank $r$. Then

$T \cdot \mu_{\tau_{+}(P)}(S, T)=\mu_{P}(S, T)+(T-1) \cdot \mu_{P}(S, 0)+S^{r-1} T-S^{r} T$.

Proof Just as in the case of the coboundary polynomial, we will use a reduced form of the polynomial to work with. Let

$\bar{\mu}_{P}(S, T)=\mu_{P}(S, T)-S^{r}=\sum_{j=0}^{r} \bar{\mu}_{j}(S) T^{r-j}$,

where

$\bar{\mu}_{j}(S)=\sum_{\substack{r_{P}(x)=i \\ i<r}} \sum_{\substack{x \leq y \\ r_{P}(y)=j}} \mu_{P}(x, y) S^{i}$. 
Notice that the sets $\left\{x \in L: a_{L}(x)<m\right\}$ and $\left\{x \in L: r_{L}(x)<r\right\}$ are the same, so the proof that the reduced Möbius polynomial obeys the constrains in Theorem 13 is exactly the same as in the case of the reduced coboundary polynomial. Hence the reduced Möbius polynomial has a truncation formula as in Theorem 13. To switch to the non-reduced Möbius polynomial, $-T S^{r-1}$ gets added at the left hand side of the formula, and $-S^{r}-(T-1) S^{r}$ at the right hand side. This gives the term $+S^{r-1} T-S^{r} T$ in the formula.

Example 17 We consider again the uniform geometric lattice $L=U_{r, n}$. For all elements $x \in U_{r, n}$ with $r_{L}(x)<$ $r(L)$, we have that $a_{L}(x)=r_{L}(x)$. Therefore the coboundary polynomial and the Möbius polynomial differ only in the leading term: for the coboundary polynomial this is $S^{n}$, while for the Möbius polynomial it is $S^{r(L)}$. For example, the Möbius polynomial of the geometric lattice $U_{3,7}$ is equal to

$\mu_{U_{3,7}}(S, T)=S^{3}+21 S^{2}(T-1)+7 S\left(T^{2}-6 T+5\right)+\left(T^{3}-7 T^{2}+21 T-15\right)$.

The Möbius polynomial of its truncation $U_{2,7}$ is equal to

$\mu_{U_{2,7}}(S, T)=S^{2}+7 S(T-1)+\left(T^{2}-7 T+6\right)$.

Comparing with Example 14, we see why the term $S^{r-1} T-S^{r} T$ is needed in the truncation formula of the Möbius polynomial.

We would like to mention that it is possible to give a general formula for the Möbius polynomial of a uniform geometric lattice; see for example $\$ 5.5$ and Proposition 51 of [8]. Checking the truncation formula directly in the case of a general uniform geometric lattice is therefore possible, but we leave the rather lengthy calculation to the reader.

For posets, we can find a similar truncation formula for the lower truncation.

Theorem 18 Let $P$ be a poset with rank function of rank $r$. Then

$S \cdot \mu_{\tau_{-}(P)}(S, T)=\mu_{P}(S, T)+(S-1) \cdot \mu_{P}(0, T)+S T^{r-1}-S T^{r}$.

Proof We prove this formula by using the inverse poset. The Möbius polynomial of the inverse poset $i(P)$ is found by interchanging the variables in the Möbius polynomial of the original poset $P$ :

$$
\begin{aligned}
\mu_{i(P)}(S, T) & =\sum_{x \in i(P)} \sum_{y \in i(P)} \mu_{i(P)}(x, y) S^{r_{i(P)}(x)} T^{r(i(P))-r_{i(P)}(y)} \\
& =\sum_{x \in P} \sum_{y \in P} \mu_{P}(y, x) S^{r-r_{P}(x)} T^{r-\left(r-r_{P}(y)\right)} \\
& =\sum_{x \in P} \sum_{y \in P} \mu_{P}(x, y) T^{r_{P}(x)} S^{r-r_{P}(y)} \\
& =\mu_{P}(T, S) .
\end{aligned}
$$

This makes it possible to derive the formula for lower truncation directly from the formula for upper truncation. We start with the formula for upper truncation in Theorem 16:

$T \cdot \mu_{\tau_{+}(P)}(S, T)=\mu_{P}(S, T)+(T-1) \cdot \mu_{P}(S, 0)+S^{r-1} T-S^{r} T$.

For the inverse of $P$, the same formula holds:

$T \cdot \mu_{\tau_{+}(i(P))}(S, T)=\mu_{i(P)}(S, T)+(T-1) \cdot \mu_{i(P)}(S, 0)+S^{r-1} T-S^{r} T$.

Now we use that $\tau_{+}(i(P))=i\left(\tau_{-}(P)\right)$ and $\mu_{i(P)}(S, T)=\mu_{P}(T, S)$ :

$T \cdot \mu_{\tau_{-}(P)}(T, S)=\mu_{P}(T, S)+(T-1) \cdot \mu_{P}(0, S)+S^{r-1} T-S^{r} T$.

Interchanging $S$ and $T$ gives the formula for lower truncation. 


\section{Truncation Formula for the Spectrum Polynomial}

The spectrum polynomial was first introduced in [9]. By a slight change of variables, we get a polynomial on which we can apply the same technique as before to prove a truncation formula.

Definition 19 Let $M$ be a matroid and $L=L(M)$ its associated geometric lattice. The spectrum polynomial of a matroid in the variables $S$ and $T$ is given by

$\operatorname{Spec}_{M}(S, T)=\sum_{x \in L} \sum_{x \leq y \in L}|\tilde{\chi}(I N(x))|\left|\mu_{L}(x, y)\right| S^{|x|} T^{r_{L}(y)}$

where $|x|$ is the number of elements of $M$ that are in the flat $x$ and $\tilde{\chi}(I N(x))$ is the reduced Euler characteristic of the independence complex of $M$.

The value of $|x|$ is equal to $a_{L}(x)$ for all flats $x$ if $M$ is a simple matroid. Furthermore, the number of elements of $M$ that are in the flat $x$ is equal to the number of elements of $\tau(M)$ that are in $x$ if $r(x) \leq r(M)-2$.

Let $\mathcal{I}_{M}$ be the collection of independent subsets of $M$. The following formula holds by [2, p. 238]:

$|\tilde{\chi}(I N(M))|=R_{M}(-1,0)=\sum_{I \in \mathcal{I}_{M}}(-1)^{r(M)-|I|}$.

Definition 20 Let $M$ be a matroid and $L=L(M)$ its associated geometric lattice. Let

$v_{M}(x, y)=(-1)^{r_{M}(x)} \mu_{L}(x, y)|\tilde{\chi}(I N(x))|$

and define the reciprocal alternating spectrum polynomial of $M$ by

$\operatorname{Rasp}_{M}(S, T)=\sum_{x \in L} \sum_{x \leq y \in L} v_{M}(x, y) S^{|x|} T^{r(L)-r_{L}(y)}$.

Notice the similarity to the definition of the coboundary polynomial. The polynomial $\operatorname{Rasp}_{M}(S, T)$ is equivalent to the spectrum polynomial.

Theorem 21 The polynomial $\operatorname{Rasp}_{M}(S, T)$ is the reciprocal alternating polynomial of $\operatorname{Spec}_{M}(S, T)$ in $T$ :

$\operatorname{Rasp}_{M}(S, T)=T^{r(M)} \operatorname{Spec}_{M}\left(S,-\frac{1}{T}\right)$.

Proof Let $L=L(M)$. Using the fact that $|\mu(x, y)|=(-1)^{r(y)-r(x)} \mu(x, y)$ and rewriting gives:

$$
\begin{aligned}
T^{r(M)} \operatorname{Spec}_{M}\left(S,-\frac{1}{T}\right) & =T^{r(M)} \sum_{x \in L} \sum_{x \leq y \in L}\left|\mu_{L}(x, y)\right||\tilde{\chi}(I N(x))| S^{|x|}\left(-T^{-1}\right)^{r(y)} \\
& =T^{r(M)} \sum_{x \in L} \sum_{x \leq y \in L}(-1)^{r(y)-r(x)} \mu_{L}(x, y)|\tilde{\chi}(I N(x))| S^{|x|}\left(-T^{-1}\right)^{r(y)} \\
& =T^{r(M)} \sum_{x \in L} \sum_{x \leq y \in L}(-1)^{r(x)} \mu_{L}(x, y)|\tilde{\chi}(I N(x))| S^{|x|}\left(T^{-1}\right)^{r(y)} \\
& =\sum_{x \in L} \sum_{x \leq y \in L} v_{M}(x, y) S^{|x|} T^{r(M)-r(y)} \\
& =\operatorname{Rasp}_{M}(S, T) .
\end{aligned}
$$

We omit the subscripts $L$ and $M$ by the rank function to emphasize the cryptomorphism between $L(M)$ and $M$. 
We need the following computational lemmas about $\operatorname{Rasp}_{M}$ and $v_{M}$.

Lemma 22 Let $\mathcal{B}_{M}$ be the collection of all bases of a matroid $M$. Then

$\operatorname{Rasp}_{M}(1,0)=(-1)^{r(M)}\left|\mathcal{B}_{M}\right|$.

Proof Let $\mathcal{I}$ be the set of independent sets of $M$, and let $L=L(M)$.

$$
\begin{aligned}
\operatorname{Rasp}_{M}(1,0) & =\sum_{x \in L} v_{M}\left(x, 1_{L}\right) \\
& =\sum_{x \in L}(-1)^{r(x)} \mu_{L}\left(x, 1_{L}\right) \sum_{\substack{I \subseteq x \\
I \in \mathcal{I}}}(-1)^{r(x)-|I|} \\
& =\sum_{I \in \mathcal{I}}(-1)^{-|I|} \sum_{\substack{I \leq x \leq 1_{L} \\
\mu_{L}}}\left(x, 1_{L}\right) \\
& =\sum_{B \in \mathcal{B}}(-1)^{-r(M)} \\
& =(-1)^{-r(M)}\left|\mathcal{B}_{M}\right|
\end{aligned}
$$

Lemma 23 Let $M$ be a matroid and $x<y$ elements of $L=L(M)$. Then $v_{M}(x, y)$ has the following induction formula:

$$
\sum_{x \leq z \leq y} v_{M}(x, z)=\sum_{x \leq z \leq y} v_{M}(z, y)=0 .
$$

Proof For $x<y$ we have

$$
\begin{aligned}
v_{M}(x, y) & =(-1)^{r_{M}(x)}|\tilde{\chi}(I N(x))| \mu_{L}(x, y) \\
& =(-1)^{r_{M}(x)}|\tilde{\chi}(I N(x))| \sum_{x \leq z<y}-\mu_{L}(x, z) \\
& =-\sum_{x \leq z<y} v_{M}(x, z) .
\end{aligned}
$$

This implies the given induction formula.

We can now prove a truncation formula for the polynomial $\operatorname{Rasp}_{M}(S, T)$.

Theorem 24 Let $M$ be a matroid of rank $r$ on a set of $m$ elements. Then

$T \cdot \operatorname{Rasp}_{\tau(M)}(S, T)=\operatorname{Rasp}_{M}(S, T)+(T-1) \cdot \operatorname{Rasp}_{M}(S, 0)-S^{m} T \cdot \operatorname{Rasp}_{M}(1,0)$.

Proof Just as in the previous cases, we will use a reduced form of the polynomial to work with. Let

$\overline{\operatorname{Rasp}}_{M}(S, T)=\operatorname{Rasp}_{M}(S, T)-v_{M}\left(1_{M}, 1_{M}\right) S^{m}=\sum_{j=0}^{r} \overline{\operatorname{Rasp}}_{j}(S) T^{r-j}$,

where

$$
\overline{\operatorname{Rasp}}_{j}(S)=\sum_{\substack{|x|=i \\ i<m}} \sum_{\substack{x \leq y \\ r_{M}(y)=j}} v_{M}(x, y) S^{i} .
$$


Notice that the sets $\{x \in M:|x|<m\}$ and $\left\{x \in M: r_{M}(x)<r\right\}$ are the same and that Lemma 23 gives the same induction formula for $v(x, y)$ as we have for $\mu(x, y)$ so again we can copy the previous proofs to show that the reduced Rasp polynomial obeys the constrains in Theorem 13 . Hence the reduced Rasp polynomial has a truncation formula as in Theorem 13. To switch to the non-reduced Rasp polynomial, $-v_{\tau(M)}\left(1_{\tau(M)}, 1_{\tau(M)}\right) S^{m} T$ gets added at the left hand side of the formula, and $-v_{M}\left(1_{M}, 1_{M}\right) S^{m}-(T-1) v_{M}\left(1_{M}, 1_{M}\right)$ at the right hand side. In total, we have to add $-S^{m} T$ times the following:

$$
\begin{aligned}
v_{M}\left(1_{M}, 1_{M}\right)-v_{\tau(M)}\left(1_{\tau(M)}, 1_{\tau(M)}\right) & =(-1)^{r} \cdot 1 \cdot \sum_{I \in \mathcal{I}_{M}}(-1)^{r-|I|}-(-1)^{r-1} \cdot 1 \cdot \sum_{I \in \mathcal{I}_{\tau(M)}}(-1)^{r-1-|I|} \\
& =(-1)^{r}\left(\sum_{I \in \mathcal{I}_{M} \backslash \mathcal{B}_{M}}(-1)^{r-|I|}+\sum_{I \in \mathcal{B}_{M}}(-1)^{r-r}+\sum_{I \in \mathcal{I}_{M} \backslash \mathcal{B}_{M}}(-1)^{r-1-|I|}\right) \\
& =(-1)^{r} \sum_{I \in \mathcal{B}_{M}} 1=(-1)^{r}\left|\mathcal{B}_{M}\right| .
\end{aligned}
$$

Combining with Lemma 22, the theorem follows.

The truncation formula for $\operatorname{Rasp}_{M}(S, T)$ also makes it possible to determine the spectrum polynomial of a truncated matroid from the spectrum polynomial of the matroid itself. However, if we try to apply Theorem 21 directly to the truncation formula for $\operatorname{Rasp}_{M}(S, T)$, we run into trouble with the terms that have $T=0$. To avoid this, notice that the term $\operatorname{Rasp}_{M}(S, 0)$ actually means "write $\operatorname{Rasp}_{M}(S, T)$ as a polynomial in $T$ and take the coefficient of $T^{0}$ ". In terms of the spectrum polynomial, this is " $\operatorname{write} \operatorname{Spec}_{M}(S, T)$ as a polynomial in $T$, take the coefficient of $T^{r(M)}$ and multiply by $(-1)^{r(M)}$ ". This motivates the following definition.

Definition 25 The polynomials $S_{i}(S)$ are the coefficients of the spectrum polynomial, written as a polynomial in $T$ :

$\operatorname{Spec}_{M}(S, T)=\sum_{i=0}^{r(M)} S_{i}(S) T^{i}$.

From this definition, it follows that we can write

$\operatorname{Rasp}_{M}(S, T)=\sum_{i=0}^{r(M)}(-1)^{i} S_{i}(S) T^{r(M)-i}$.

We use this to prove the truncation formula for the spectrum polynomial.

Theorem 26 Let $M$ be a matroid of rank $r$ on a set of $m$ elements. Then

$\operatorname{Spec}_{\tau(M)}(S, T)=\operatorname{Spec}_{M}(S, T)-T^{r-1}(T+1) \cdot S_{r}(S)+S^{m} T^{r-1} \cdot S_{r}(1)$.

Proof The truncation formula for $\operatorname{Rasp}_{M}(S, T)$ can be written as

$T \cdot \operatorname{Rasp}_{\tau(M)}(S, T)=\operatorname{Rasp}_{M}(S, T)+(-1)^{r}(T-1) \cdot S_{r}(S)-(-1)^{r} S^{m} T \cdot S_{r}(1)$.

Using the equivalence in Theorem 21, we get

$T \cdot T^{r-1} \cdot \operatorname{Spec}_{\tau(M)}\left(S,-\frac{1}{T}\right)=T^{r} \cdot \operatorname{Spec}_{M}\left(S,-\frac{1}{T}\right)+(-1)^{r}(T-1) \cdot S_{r}(S)-(-1)^{r} S^{m} T \cdot S_{r}(1)$.

Applying the transformation $T \rightarrow-\frac{1}{T}$ and multiplying by $(-T)^{r}$ gives the desired truncation formula for the spectrum polynomial. 
Example 27 In Examples 14 and 17 we showed truncation formulas for the uniform matroids $U_{r, n}$. For the spectrum polynomial, we will not give a general formula, but demonstrate the truncation formula by mentioning

$$
\begin{aligned}
& \operatorname{Rasp}_{U_{3,7}}(S, T)=T^{3}-7 T^{2}+21 T-15-20 S^{7} \\
& \operatorname{Spec}_{U_{3,7}}(S, T)=20 T^{3} S^{7}+15 T^{3}+21 T^{2}+7 T+1
\end{aligned}
$$

and for the truncation

$$
\begin{aligned}
& \operatorname{Rasp}_{U_{2,7}}(S, T)=T^{2}-7 T+6+15 S^{7} \\
& \operatorname{Spec}_{U_{2,7}}(S, T)=15 T^{2} S^{7}+6 T^{2}+7 T+1 .
\end{aligned}
$$

\section{Applications and Generalizations}

We have given truncation formulas for the coboundary and Möbius polynomial of a geometric lattice and the spectrum polynomial of a matroid, generalizing the truncation formula of the rank generating polynomial of a matroid and the characteristic polynomial of a geometric lattice. These formulas trigger a lot of other questions about their application and possible generalizations. The following list is by no means exhaustive, but it serves as encouragement and inspiration for further research.

There are several constructions on matroids closely related to the ordinary truncation, for example the previously mentioned Dilworth truncation, the principal truncation, and weak and strong maps; see [4,7,10,11]. It would be interesting to see whether truncation-like formulas also exist for these constructions.

Matroids representable over a finite field are closely related to linear codes. We showed that the truncation of a matroid $M$ that is representable over a finite field is representable over a finite extension of the same finite field. We gave an upper bound for the extension degree that is needed. However, in practice it is possible that $\tau(M)$ is already representable over a much smaller extension field. By using the extended weight enumerator of linear codes (see [8]), a polynomial that is equivalent to the rank polynomial, it might be possible to find better bounds for the representation of $\tau(M)$.

The Möbius polynomial determines the Whitney numbers of the first and second kind. These sequences for geometric lattices are conjectured to be unimodal, by Rota [15], and even log-concave, by Welsh [17]. A recent overview of the progression on the conjectures can be found in $[14, \S 15.2]$.

The truncation formula for the Möbius polynomial gives relations between the Whitney numbers of a poset with rank function $P$ and the Whitney numbers of its truncations $\tau_{-}(P)$ and $\tau_{+}(P)$. This gives an approach to the proof of the unimodal conjectures by induction.

We saw that the lower truncation of a geometric lattice does not need to be a geometric lattice. Also, the unimodal conjectures are not true for posets with rank function in general. So the challenge is to find a class of objects that is "in between" posets with rank function and geometric lattices: this class needs to be closed under lower truncation, while still the unimodal property holds. Also, for the induction to work, the smallest cases in this class need to be proved unimodal.

The spectrum polynomial is in general not determined by the rank generating polynomial of a matroid; see [9]. In order to find counterexamples for the implication in the other direction, we see from the truncation formulas for the coboundary and rasp polynomial that it is sufficient to find a counterexample with respect to the one variable polynomials in $S$ setting $T=0$.

Acknowledgments We like to thanks the referees for the detailed remarks and the suggestion to give shorter proofs of the three main theorems.

Open Access This article is distributed under the terms of the Creative Commons Attribution License which permits any use, distribution, and reproduction in any medium, provided the original author(s) and the source are credited. 


\section{References}

1. Aigner, M. : Combinatorial Theory. Springer, New York (1979)

2. Björner, A.: The homology and shallability of matroids and geometric lattices. In: White, N. (ed.) Matroid Applications, pp. 226-280. Cambridge University Press, Cambridge (1992)

3. Britz, T. : Higher support matroids. Discrete Math. 307, 2300-2308 (2007)

4. Brylawski, TH: Constructions. In: White, N. (ed.) Theory of Matroids, pp. 127-223. Cambridge University Press, Cambridge (1986)

5. Crapo, H.: Möbius inversion in lattices. Archiv der Mathematik 19, 595-607 (1968)

6. Crapo, H.: The Tutte polynomial. Aequationes Mathematicae 3, 211-229 (1969)

7. Dilworth, R.: Dependence relations in a semimodular lattice. Duke Math. J. 11, 575-587 (1944)

8. Jurrius, R.P.M.J., Pellikaan, R.: Codes, arrangements and matroids. In: Martinez-Moro, E. (ed.) Algebraic Geometry Modeling in Information Theory Cryptography, vol. 9. Series on coding theory and cryptology, World Scientific Publishing (2012)

9. Kook, W., Reiner, V., Stanton, D.: Combinatorial laplacians of matroid complexes. J. Am. Math. Soc. 13, 129-148 (2000)

10. Kung, J.P.S.: Strong maps. In: White, N. (ed.) Theory of Matroids, pp. 224-253. Cambridge University Press, Cambridge (1986)

11. Kung, J.P.S., Nguyen, H.Q.: Weak maps. In: White, N. (ed.) Theory of Matroids, pp. 254-271. Cambridge University Press, Cambridge (1986)

12. Lang, S.: Algebra. Addison-Wesley, Reading (1965)

13. Mason, J.H.: Matroids as the study of geometrical configurations. In: Aigner, M. (ed.) Higher Combinatorics, pp. 133-176. Reidel Publ. Comp., Dordrecht (1977)

14. Oxley, J.G.: Matroid Theory, 2nd edn. Oxford University Press, Oxford (2011)

15. Rota, G.C.: Combinatorial theory, old and new. In: Proceedings of the International Congress on Mathematics 1970 (Nice), vol. 3 , pp. 229-233, Gauthier-Villars, Paris (1971)

16. Stanley, R.P.: An introduction to hyperplane arrangements. In: Geometric Combinatorics, IAS/Park City Mathematics Series, vol. 13, pp. 389-496. American Mathematical Society, Providence, RI (2007)

17. Welsh, D.J.A.: Combinatorial problems in matroid theory. In: Welsh, D.J.A. (ed.) Combinatorial Mathematics and Its Applications, pp. 291-306. Academic Press, London (1972)

18. Welsh, D.J.A.: Matroid Theory. Academic Press, London (1976)

19. Welsh, D.J.A.: Matroids: fundamental concepts. In: Graham, R.L., Grötschel, M., Lovász, L. (eds.) Handbook of Combinatorics, vol. 1, pp. 483-526. Elsevier Scientific Publishers, Amsterdam (1995)

20. White, N.: Theory of matroids. In: Encyclopedia of Mathmatics and Its Applications, vol. 26. Cambridge University Press, Cambridge (1986) 